\title{
Radio Frequency Energy Harvesting for Low Power Sensors
}

\author{
Okan OZGONENEL*, Seda USTUN ERCAN
}

\begin{abstract}
Wireless sensor networks and the internet of things are benefiting from recent advances in power consumption to implement intelligent control entities. Similar advances in battery technology have enabled these systems to become autonomous. Nevertheless, this approach is insufficient for modern applications. An alternative solution to power these sensors is to use the energy available in their environment, such as thermal, mechanical vibration, light or radio frequencies. However, sensors are frequently placed in an environment where power density is low. This study investigates energy harvesting from radio frequencies compared to other sources. After demonstrating the potential for collecting energy over a wide frequency band, a statistical study was carried out to determine the RF power density present in the urban environment and in rural areas. Multi-band RF harvester systems were designed to harvest energy in several frequency bands to show when multiple RF sources are available. The amount of energy harvested can be increased when the system is designed to operate over a wide frequency band. In this study, multiband RF energy harvester to power wireless sensors is produced using Advanced Design Software (ADS). According to the design outcomes the proposed energy harvesting scheme works better on the GSM900 and GSM1800 bands.
\end{abstract}

Keywords: energy harvester; wireless network; wireless power

\section{INTRODUCTION}

Nowadays, the need to be able to monitor the environment in which we find ourselves is increasingly important and this allows us to manage our actions; a typical example is the weather forecast. Modern sensors are small, self-contained devices that perform simple measurements on their immediate environment. They are used to observe many physical phenomena such as temperature, pressure, brightness, etc., which is essential for many industrial and scientific applications. The role of a sensor is to transform a physical quantity into an exploitable electrical quantity, for example a digital signal usable by a computer. The interface can be made by a wired link or wirelessly, as has been the case for number of years.

At the same time, recent progress in microelectronics and micromechanics has made it possible to produce components of a few cubic millimetres in volume at reasonable cost with ever decreasing power requirements. Micro-sensors can be made as a complete embedded system and several of them are deployed to collect and transmit environmental data to one or more collection points in an autonomous manner to form a wireless sensor network (WSN). The traditional way to power these sensors is to use a battery that has a limited amount of energy and requires replacement when depleted. The maintenance related to changing batteries can be expensive, especially in the case of sensors located in locations difficult to access. An alternative form of selfpowering would be advantageous in these circumstances, and energy harvesting offers the potential.

\subsection{Energy Harvesting}

An energy harvesting system for powering a sensor is composed of five distinct blocks as shown in Fig. 1.

The first stage of the system is the energy transducer. This provides as output a physical quantity that can be used as input to the energy conversion stage. The principle for operation of the transducer is based on physical or chemical effects. There are six main classes: thermal, mechanical, optical, magnetic, electrical and chemical [1].

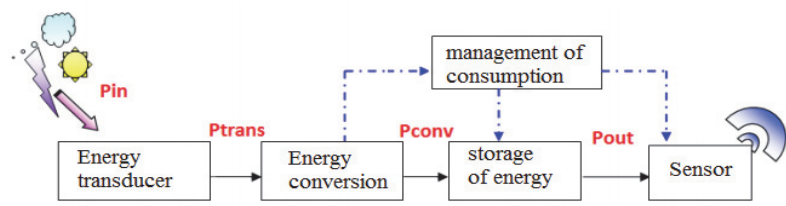

Figure 1 Block Diagram of Energy Harvesting Unit for Power Supply to an autonomous sensor

The second stage performs the conversion of the input energy into electrical power. This stage may include rectifiers, converters or charge pumps depending on the form of the input transducer. Transducers largely fall into two groups; mechanical vibration and electromagnetic waves, each generates an alternating voltage that requires the energy converter to include a voltage rectifier. Photovoltaic and thermal energy transducers may require dc-dc converter to provide a useable output voltage. The major challenge is to ensure the best conversion efficiency between the transducer and the sensor to be powered [2].

The third stage of the system is a cell to accumulate received energy and to supply this to the sensor as required [3]. This can be of various forms including electric capacitors, super capacitors or rechargeable batteries.

The fourth stage of the system manages the consumption of the energy and includes a microprocessor to implement an algorithm to control the optimum flow of energy between the storage cell and the sensor [4]. "Consumption Management", also termed "Power Management" in the literature, may also refer to the need to match the impedance of a generator to the load to provide maximum power transfer. This is generally called Maximum Power Point Tracking (MPPT) that could include a step-up voltage converter between the generator and the storage element. Research on maximum recoverable power of an energy source and is a widespread subject in the scientific literature. For example, $[4,5]$ show that the harvested energy in communicating sensors networks evolves over time and is not the same for each node of the network. The real-time consumption management algorithm therefore uses an energy prediction algorithm to determine the available energy at each time 
step and manage the load by adapting its consumption to available resources.

\section{MATERIAL AND METHODS}

In this study, two multiband RF energy harvesters are designed. In the first part, the architecture and the separate parts of the rectenna (rectifier-antenna) are selected and explained. The first rectenna was designed to harvest energy in a wide frequency band from $1.8 \mathrm{GHz}$ to $2.6 \mathrm{GHz}$. A study was then undertaken on the contribution of the separate RF branches of the rectenna in order to improve the conversion of RF to DC in a multiband rectenna. Based on the results from the first architecture, a second rectenna is presented that is designed to operate on the four frequency bands: GSM900, GSM1800, UMTS and Wi-Fi.

\subsection{Proposed Overall Architecture}

Fig. 2 shows the architecture of a multiband rectenna that includes the impedance matching network, rectifier and dc filter that constitute each RF branch. The number of such RF branches of the rectenna architecture is not limited. A single broadband receiving antenna is used as this type of antenna provides a more compact structure than multiple tuned antennas as input. RF-DC conversion efficiency is optimised by considering the impedance matching of the receiving antenna to the rectifiers and other circuit elements, and in this design, each rectifier has been matched at the selected frequency by using a bandpass impedance matching network. The bandwidth has been chosen to cover the entire spectrum of RF power density in a standard RF band such as GSM900, GSM1800, or other. The low-pass filter removes the RF signals to produce a DC component to pass to the output load. Finally, the outputs of the RF branches are connected together to sum the contributions.

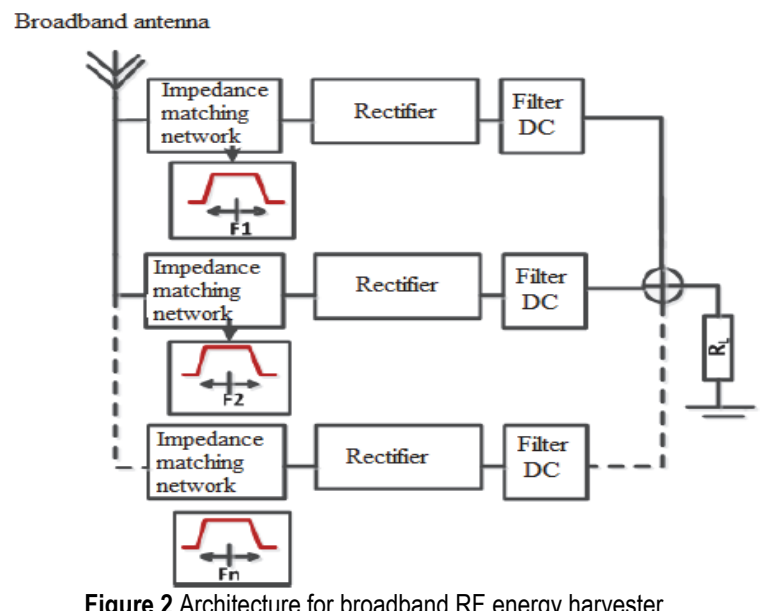

\subsection{Choice of Rectifier and Schottky Diode}

Energy harvesting from the environment often produces low levels of power with small amplitude. In order to minimise power loss in the rectifier, devices with low threshold voltage, $\mathrm{V}_{\mathrm{TH}}$, must be used [6]. The normal solution to overcome this problem is to use Schottky diodes with low $\mathrm{V}_{\mathrm{TH}}$, sometimes called "zero bias" diodes. In the literature, many prototypes are designed with the Schottky diode SMS7630 [7] that has a threshold voltage of $150 \mathrm{mV}$. A diode with similar $V_{\mathrm{TH}}$ is the MSS20-141 diode, which is used in commercial dosimeters. The parameters of the two diodes are given in Tab. 1. The internal resistance, $R_{\mathrm{s}}$, for these diodes is quite high, of the order of $20 \Omega$ and depends mainly on the diode technology, such as the metal used, the $N+$ doping assay and the surface of the diode. The MSS20-141-B01D has been selected for this work.

The conversion efficiency, $\eta$, is defined as:

$\eta=\frac{P_{d c}}{P_{i n}}$

where $P_{d c}$ is the electrical output power from the energy converter and $P_{\text {in }}$ is the RF input power.

Table 1 Schottky Diode SMS7630 and MSS20-141

\begin{tabular}{|c|c|c|c|c|}
\hline Parameters & Description & SMS7630 & MSS20-141 & Unit \\
\hline$B_{\mathrm{v}}$ & $\begin{array}{c}\text { Reverse } \\
\text { breakdown voltage }\end{array}$ & 2 & 7.0 & $\mathrm{~V}$ \\
\hline$C_{\mathrm{j} 0}$ & $\begin{array}{c}\text { Junction } \\
\text { capacitance at zero } \\
\text { bias }\end{array}$ & 0.14 & 0.08 & $\mathrm{pF}$ \\
\hline$E_{\mathrm{g}}$ & Band-gap energy & 0.069 & 0.069 & $\mathrm{eV}$ \\
\hline$I_{\mathrm{s}}$ & $\begin{array}{l}\text { Reverse saturation } \\
\text { current }\end{array}$ & 5 & 3.6 & $\mu \mathrm{A}$ \\
\hline$N$ & Emission Factor & 1.05 & 1.25 & \\
\hline$R_{\mathrm{s}}$ & $\begin{array}{l}\text { Parasitic series } \\
\text { resistance }\end{array}$ & 20 & 20 & $\Omega$ \\
\hline$P_{\mathrm{b}}(\mathrm{VJ})$ & Junction potential & 0.3 & 0.15 & V \\
\hline$P_{\mathrm{t}}(\mathrm{XTI})$ & $\begin{array}{l}\text { Saturation current } \\
\text { temperature } \\
\text { exponent }\end{array}$ & 2 & 2 & \\
\hline$M$ & $\begin{array}{c}\text { Junction } \\
\text { coefficient of } \\
\text { variation }\end{array}$ & 0.4 & 0.5 & \\
\hline
\end{tabular}

\subsection{Design of the Impedance Matching Network}

Maximum power is transferred to a load when the complex impedance of the source is equal to the complex impedance of the load and associated components. The most frequently used impedance adaptation networks are the $L, \Pi$ or $T$ [8]. The quality factor, $Q$, which is the ratio between the energy stored in the network and the energy dissipated by the network, is an important parameter; a high value of $Q$ means a small amount of energy is dissipated but also implies a narrow bandwidth.

One RF branch of the rectenna is shown in Fig. 3. The receiving antenna is modelled by a simple resistor $\mathrm{Ra}$. The rectifier is the series rectifier associated with an $\mathrm{LC}$ filter, which acts as a dc filter. A resistor, RL, of $430 \Omega$, models the average equivalent impedance for several sensors, as found in the scientific literature [9]. To design the impedance matching network, it is first necessary to determine the input impedance of the rectifier, Zrect.

$\Pi$ or $T$ networks provide the greatest flexibility to the designer and provide better adaptation over a given frequency band. If it is assumed that the impedance of the antenna is $50 \Omega$ for all frequencies, then the input impedance of the rectifier is always lower than that of the antenna. The most suitable network for matching in these circumstances is the $T$ network. 


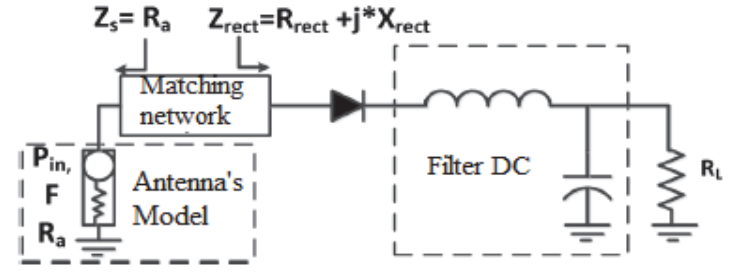

Figure 3 An RF Branch of the modelled RF energy harvester

The proposed adaptation network is shown in Fig. 4. In order to increase the bandwidth of the network and to eliminate low frequencies, an inductor $L_{3}$ is added in parallel with the capacitor of the $T$-filter.

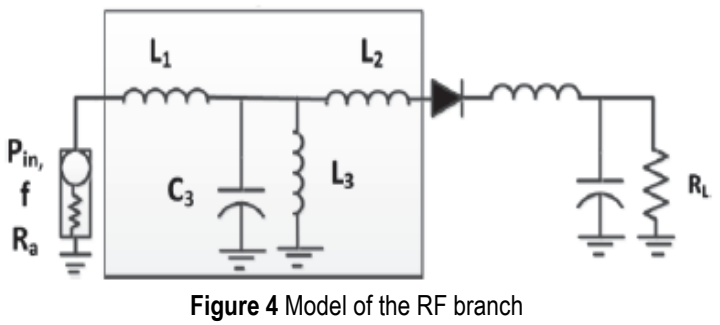

The impedance matching network therefore comprises four components. As the frequency and bandwidth of operation may take on a wide range of values, an optimization process is necessary to determine component values for a specific case.

The objective is to calculate the values of the matching network components such that the maximum power is transferred from the receiving antenna to the load in the selected RF band. Considering the circuit of Fig. 5, the impedance of the antenna seen by the matching network is equal to $R_{\mathrm{a}}$. However, Fig. 5 now includes the impedance of the rectifier, modelled as $Z_{\text {rect }}=R_{\text {rect }}+j X_{\text {rect. }}$.

The voltage transfer function, $H(\omega, Q)$, can be expressed as a function of the quality factor of the adaptation network, $Q$ (2). In addition, network components are expressed as a function of $Q(3-7)$. It should be noted that $L_{3}$ (as lumped parameter) depends on the bandwidth of the adaptation network, which is a further degree of freedom in the design.

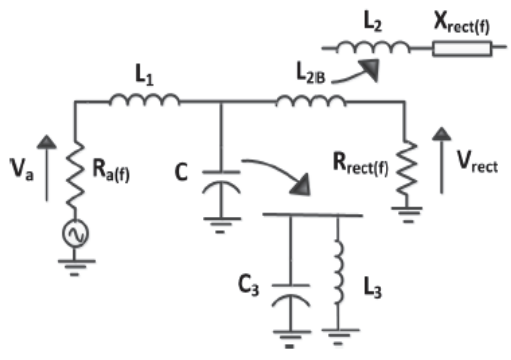

Figure 5 Modelling the impedance matching network

$H(\omega, Q)=\frac{2\left(\frac{R_{\text {rect }}}{R_{\mathrm{a}}}+1\right) Q-\left(\frac{4 Q^{2} R_{\text {rect }}}{R_{\mathrm{a}}}-\left(\frac{R_{\mathrm{rect}}}{R_{\mathrm{a}}}-1\right)^{2}\right)}{\{A+B\}^{\frac{1}{2}}}$

where:

$$
\begin{aligned}
& A=4\left[\left(\frac{R_{\text {rect }}}{R_{\mathrm{a}}}+1\right)^{2} Q-\left(\frac{R_{\text {rect }}}{R_{\mathrm{a}}}+1\right)\left(\frac{4 Q^{2} R_{\text {rect }}}{R_{\mathrm{a}}}-\left(\frac{R_{\text {rect }}}{R_{\mathrm{a}}}-1\right)^{2}\right)-\right. \\
& \left.-\left(\frac{R_{\mathrm{rect}}}{R_{\mathrm{a}}}-1\right)^{2} Q \beta \omega_{P}^{2}\right]^{2}
\end{aligned}
$$

$$
\begin{aligned}
& B=\left[\left(\frac{R_{\text {rect }}}{R_{\mathrm{a}}}-1\right)^{2}\left(3 \omega_{P}-\omega_{P}{ }^{3}\right)+\left(2\left(\frac{R_{\text {rect }}}{R_{\mathrm{a}}}+1\right) Q-\right.\right. \\
& \left.-\left(\frac{8 Q^{2} R_{\text {rect }}}{R_{\mathrm{a}}}\right)\left(3 \omega_{P}-\omega_{P}{ }^{3}\right)\right]^{2}
\end{aligned}
$$

For $\omega_{P}=\omega / \omega_{C}$ and $Q=\frac{1}{2}\left(\frac{X_{L 1}}{R_{\mathrm{a}}}+\frac{X_{L 1 B}}{R_{\text {rect }}}\right)$

$L_{1}=\frac{R_{\text {rect }}\left(2 Q_{O}-\sqrt{\frac{4 Q_{O}^{2} R_{\text {rect }}}{R_{\mathrm{a}}}-\left(\frac{R_{\text {rect }}}{R_{\mathrm{a}}}-1\right)^{2}}\right)}{\omega_{C}\left(1-\frac{R_{\text {rect }}}{R_{\mathrm{a}}}\right)^{2}}$

$L_{2}=\frac{R_{\text {rect }}\left(2 Q_{O} \frac{R_{\text {rect }}}{R_{\mathrm{a}}}-\sqrt{\frac{4 Q_{O}^{2} R_{\text {rect }}}{R_{\mathrm{a}}}-\left(\frac{R_{\text {rect }}}{R_{\mathrm{a}}}-1\right)^{2}}\right)}{\omega_{C}\left(1-\frac{R_{\text {rect }}}{R_{\mathrm{a}}}\right)^{2}}-X_{\text {rect }}$

$C_{3}=\frac{2 Q_{O}}{\omega_{C} R_{\text {rect }}\left(1+\left(\frac{L_{1}}{R_{\mathrm{a}}}\right)^{2}\right)}$

$L_{3}=\frac{B_{\text {width }}}{4 \pi^{2} C_{3} F_{s}^{2}}$

Using these equations, values for the components for adaptation networks with two frequencies were calculated, as given in Tab. 2. In order to illustrate the approach, the circuit of Fig. 6 was simulated for different bandwidths to match a load impedance of $Z_{\mathrm{L}}=20+j 250 \Omega$ with $R_{\mathrm{a}}=50$ $\Omega . Z_{\mathrm{L}}$ is an impedance of a sensor to be fed.

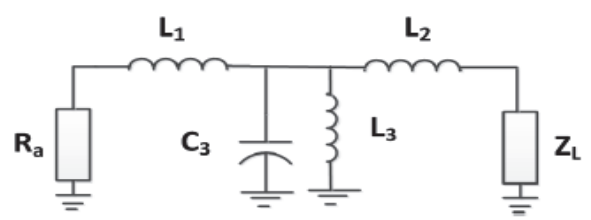

Figure 6 Simulated network diagram under ADS

Table 2 Component values for impedance matching

\begin{tabular}{|c|c|c|c|c|c|}
\hline Network & $\begin{array}{c}L_{1} / \\
\mathrm{nH}\end{array}$ & $\begin{array}{c}L_{2} / \\
\mathrm{nH}\end{array}$ & $\begin{array}{c}L_{3} / \\
\mathrm{pF}\end{array}$ & $\begin{array}{c}C_{3} / \\
\mathrm{pF}\end{array}$ & $\begin{array}{c}\text { Frequency } / \\
\mathrm{MHz}\end{array}$ \\
\hline 1 & 10 & 12 & 10.5 & 0.5 & 350 \\
\hline 2 & 11 & 10 & 12.5 & 0.5 & 250 \\
\hline
\end{tabular}


The effectiveness of the proposed network, which may be considered as the combination of two $L$-shaped networks, will depend on the quality factor of individual components used for the network. As the load impedance $\left(Z_{\mathrm{L}}\right)$ to be adapted is moved away from $50 \Omega$, the higher will be the insertion loss, which is estimated to be $2-3 \mathrm{~dB}$ in computer simulations for quality factors in the order of 30 .

To reduce these losses, it would be necessary to use high quality components with low $Q$ coefficient (such as the use of MEMS for example) or replace some components with distributed elements.

\subsection{Design of the Multiband Prototype}

The prototype has been designed to provide adaptation for three frequency bands: GSM1800, UMTS and Wi-Fi. The GSM1800 frequency band extends from 1710 to 1880 $\mathrm{MHz}$ (including the Down Link and the Up Link), that of UMTS from 1920 to $2170 \mathrm{MHz}$ (including the Down Link and the Up Link) and that of Wi-Fi from 2.41 to $2.48 \mathrm{GHz}$. These bands were selected because they are the predominant frequencies in an urban environment. The circuit of the rectenna is shown in Fig. 7. The selected Schottky diode is the Metelics MSS20-141 diode. The output dc filter comprises an inductor of $3.3 \mathrm{nH}$ and a capacitor of $68 \mathrm{pF}$, with values being determined by simulation to obtain a DC output voltage, input voltage of sensor. The antenna impedance, $\mathrm{Ra}$, is set at $50 \Omega$ for all three RF bands. The load is a resistance of $430 \Omega$. The circuit was simulated using the simulation software ADS [10] using Harmonic Balance Mode.

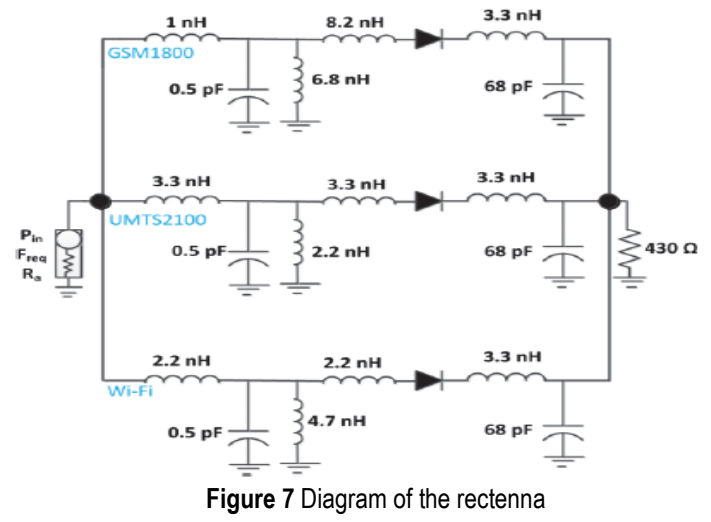

The equations permit a first estimate of the values of the network components to be made, but these may not correspond to standard component values. Values may need to be adjusted, and the Tuning function of the Advanced Design System may be used. Advanced Design System may also be used to change one or more design parameter values to see its effect on the output without need to re-simulate the entire design. The final values of the components of the three networks are shown in Fig. 7. The output voltage of the rectenna using these component values was simulated as a function of frequency and the result is shown in Fig. 8. The circuit was designed to cover frequency band ranges from 1.8 to $2.6 \mathrm{GHz}$. As the three frequency bands have significant overlap, the circuit acts as a single broadband network, as shown in Fig. 8.
The inductors and capacitors can be realized as localized or distributed technology; the choice depends on the working frequency, the values of the components, and the desired performance [11].

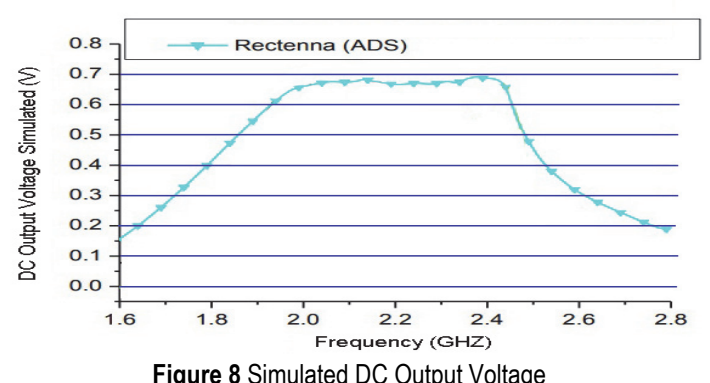

The maximum output voltage of $0.6 \mathrm{~V}$ is achieved at $2.4 \mathrm{GHz}$ for an input power of $-20 \mathrm{dBm}$ for the $430 \Omega$ load. The efficiency is calculated from the output voltage using the following equation:

$\eta(\%)=\frac{V_{\text {out }}^{2}}{R_{\mathrm{L}} \cdot P_{\text {in }}} \times 100$

where $P_{\text {in }}$ is the applied RF signal in $\mathrm{mW}, V_{\text {out }}$ is the output DC voltage, and $R_{\mathrm{L}}$ is the load.

The maximum efficiency of $40 \%$ is achieved at the operating frequencies $1.8 \mathrm{GHz}, 2.1 \mathrm{GHz}$ and $2.4 \mathrm{GHz}$ for a total input power of $-20 \mathrm{dBm}$.

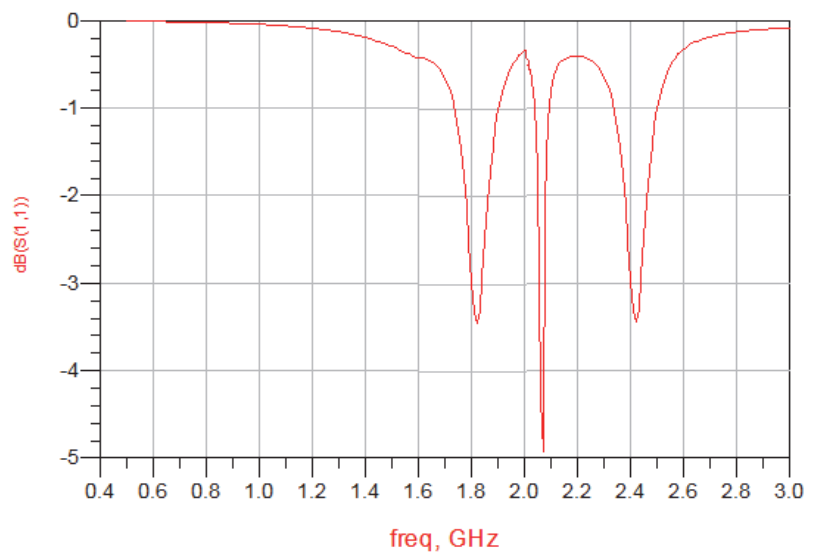

Figure 9 Reflection coefficient with respect to frequency from simulation

The rectenna works well at $1.8 \mathrm{GHz}, 2.1 \mathrm{GHz}$ and 2.4 $\mathrm{GHz}$ where $S_{11}=-3.5 \mathrm{~dB}$ and $-5 \mathrm{~dB}$ (Fig. 9). At other frequencies the rectenna performance will be negligible as $\mathrm{S}_{11}$ is close to $0 \mathrm{~dB}$ as all the power is reflected. Although this design of rectenna makes it possible to harvest energy from several RF bands and proves satisfactory for providing power to small sensors, nevertheless, a way to improve the summation of the contributions from the three RF branches has been studied. Indeed, the method of summation of several RF branches can directly affect the overall efficiency of the rectenna [12]. The DC contribution of several rectifiers can be summed in two different ways, either in parallel (current summing) (Fig. 10a) or series (voltage summing) (Fig. 10b).

The total power harvested with parallel summation will be lower than that of series summation $[13,14]$ as only 
the contribution from the branch with the highest voltage will contribute to the current at any given time and no power is taken from the other branches. With series summation, each branch provides its full contribution to the total voltage output.

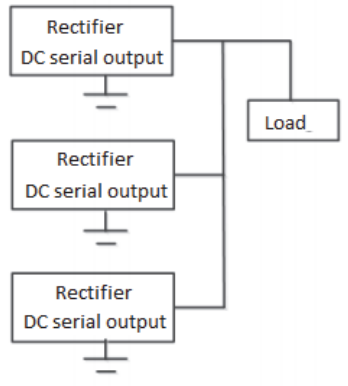

(a).

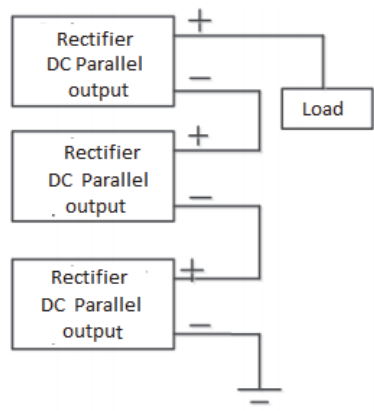

(b).
Figure 10 Options for summing DC contributions: (a) parallel connection, (b) series connection

To illustrate this point, the overall efficiency of the rectenna is studied according to the state of the three RF branches. The objective is to see the impact of an inactive branch and thus determine the effect between branches. The RF circuit shown in Fig. 11 is used for ADS simulations. If the Wi-Fi branch, UMTS or GSM1800 branch is idle, i.e. the incident power is set to $-50 \mathrm{dBm}$. For the other two active branches, the incident power varies between -20 and $0 \mathrm{dBm}$.

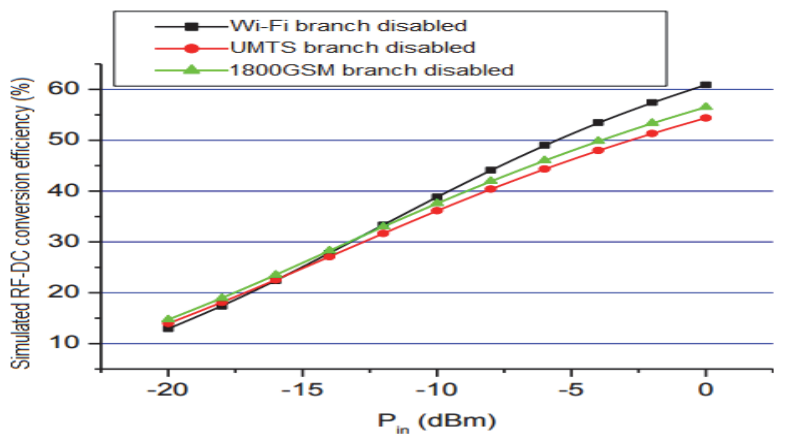

Figure 11 Simulated RF-DC Conversion Efficiency versus Power Incident $P_{\text {in }}$ for a disabled RF branch (Wi-Fi, UMTS or GSM1800)

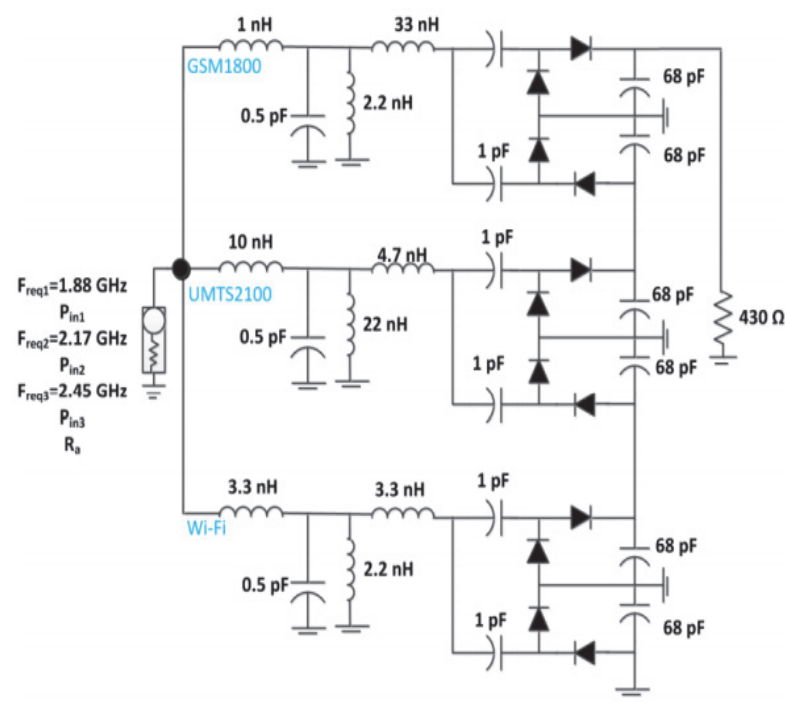

Figure 12 Rectenna with Greinacher Rectifiers
As series connection has been found to be the most efficient, the Greinacher rectifier has been selected to combine the outputs of the branches (Fig. 12). The Greinacher circuit rectifies both the positive and negative half cycles with a single diode voltage drop each half cycle and so provides greater efficiency over full-wave rectification and half-wave only. Moreover, if an RF branch is inactive, it is bypassed by DC, and it does not affect operation of the entire circuit.

For the other two active branches, the incident power varies between $-20 \mathrm{dBm}$ and $0 \mathrm{dBm}$. As shown in Fig. 14, the RF-DC conversion efficiency is not affected if a branch is disabled, regardless of the branch.

\subsection{Rectenna Architecture with Proposed Rectifier}

The architecture of the rectenna with i RF branches is shown in Fig. 13, each having a modified rectifier as shown in Fig. 13.

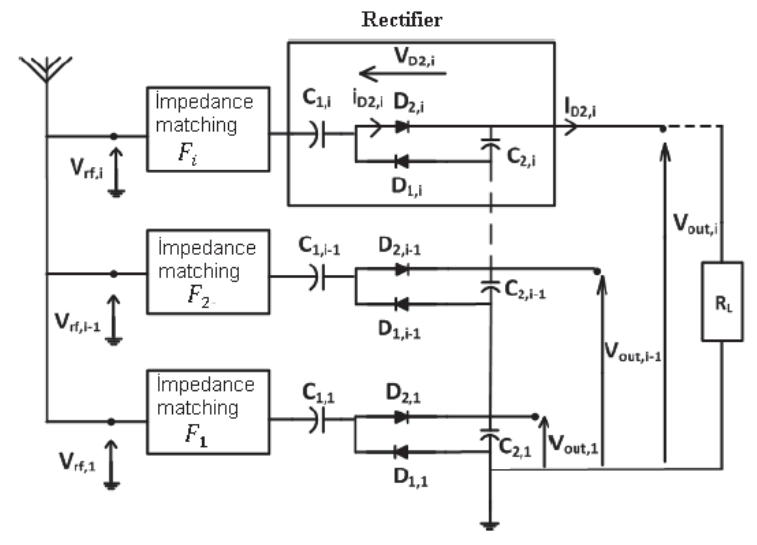

Figure 13 Rectenna architecture with the proposed rectifier

The first RF branch is referenced to ground and the ith $\mathrm{RF}$ branch is referenced to the $(i-1)$ th RF branch. The load, RL, is connected between the $i$-th RF branch and ground.

The remainder of this section shows that the voltage across the load, $V_{\text {out }}$, is the sum of the input voltages, $V_{\mathrm{rf}, \mathrm{i}}$.

$V_{D_{2, i}}=-\frac{1}{2}\left(V_{\text {out }, i}-V_{\text {out }, i-1}\right)-R_{S} I_{D_{2, i}}+\left|V_{\mathrm{rf}, i}\right| \cos (\omega t)$

where $R_{s}$ is the internal series resistance of the diode $D_{2, i}$, $I_{D_{2, i}}$ is the DC current at the output of the circuit. The current in the Schottky $D_{2, i}$ diode when conducting is:

$I_{D_{2, i}}=I_{S} \exp \left(\frac{V_{D_{2, i}}}{n V_{T}}\right)$

where $V_{T}$ is the thermodynamic voltage, $I_{S}$ is the saturation current of the diode and $n$ the factor of ideality.

From Bessel's function:

$\exp (x \cos (\omega t))=B_{0}+2 B_{k}(x) \cos (k \omega t)$

The current $I_{D 2, i}$ can be expressed by: 
$I_{D_{2, i}}=I_{s}\left[B_{0}\left(\frac{\left|V_{r f, i}\right|}{n V_{T}}\right)+2 B_{k}\left(\frac{\left|V_{r f, i}\right|}{n V_{T}}\right) \cos (k \omega t)\right] \exp (y)$

With $B_{0}$ and $y$ defined as Eq. (13) and Eq. (14):

$$
\begin{aligned}
& B_{0}(x)=\frac{\exp (x)}{\sqrt{2 \pi x}} \\
& y=\frac{-\frac{1}{2}\left(V_{\mathrm{out}, i}-V_{\mathrm{out}, i-1}\right)-R_{s} I_{D_{2, i}}}{n V_{T}}
\end{aligned}
$$

The DC current of $I_{D 2, i}$ can then be extracted:

$$
I_{D_{2, i}} \approx I_{s}\left[\exp \left[\frac{-\frac{1}{2}\left(V_{\text {out }, i}-V_{\text {out }, i-1}\right)-R_{s} I_{D_{2, i}}}{n V_{T}}\right] \frac{\exp \left[\frac{\left|V_{r f, i}\right|}{n V_{T}}\right]}{\sqrt{2 \pi \frac{\left|V_{r f, i}\right|}{n V_{T}}}}\right]
$$

From Eq. (9), we can deduce:

$$
\begin{aligned}
& \ln \left(\frac{I_{D_{2, i}}}{I_{s}}\right)=-\frac{1}{2 n V_{T}}\left(V_{\text {out }, i}-V_{\text {out }, i-1}\right)-\frac{R_{s} I_{D_{2, i}}}{n V_{T}}+\frac{\left|V_{r f, i}\right|}{n V_{T}}- \\
& -\frac{1}{2} \ln \left(2 \pi \frac{\left|V_{r f, i}\right|}{n V_{T}}\right) \\
& \left(\frac{1}{2}+\frac{R_{s}}{R_{L}}\right) V_{\text {out }, i}+n V_{T} \ln \left(\frac{V_{\text {out }, i}}{R_{s} I_{s}}\right)=\frac{1}{2} V_{\text {out }, i-1}+\left|V_{r f, i}\right|- \\
& -\frac{n V_{T}}{2} \ln \left(\frac{2 \pi\left|V_{r f, i}\right|}{n V_{T}}\right)
\end{aligned}
$$

The characteristics of the chosen Schottky diode make it possible to simplify Eq. (11). Recall that the MSS20-141 diode was chosen according to its characteristics (threshold voltage, $V_{T H}=150 \mathrm{mV}$ and junction capacitance $C j=0.08$ $\mathrm{pF}$ ). The saturation current of the diode, $I_{S}$, is $3.5 \mu \mathrm{A}$ and its internal resistance, $R_{S}$, is $20 \Omega$. The ideality factor, $n$, typically varies from 1 to 2 depending on the manufacturing process. This factor accounts for the recombination of the charge carriers as they pass through the depletion region. For the MSS20-141diode, in the depletion region, $\mathrm{n}$ is equal to 1 . If the diode is biased close to its threshold voltage, then $n$ is 1.3.

Under these conditions, assuming that $R_{L}=11 \mathrm{k} \Omega$, $\left(R_{S} / R_{L}\right)$ is equal to $1.8 \mathrm{~m} \Omega$, which makes $\left(R_{S} / R_{L}\right)$ negligible compared to $1 / 2$. Moreover, $n V_{T} \ln \left(V_{\text {out }} /\left(R_{S} / R_{L}\right)\right)$ is always negligible compared to $V_{\text {out }, i} / 2$. So Eq. (11) can be simplified as Eq. (18):

$$
\frac{1}{2} V_{\mathrm{out}, i}=\frac{1}{2} V_{\text {out }, i-1}+\left|V_{r f, i}\right|-\frac{n V_{T}}{2} \ln \left(\frac{2 \pi\left|V_{r f, i}\right|}{n V_{T}}\right)
$$

If the operation is repeated, $V_{\text {out }, i}$ can be expressed as a function of the incident RF signals, $\left|V_{r f, i}\right|$ :

$$
V_{\text {out }, i}=\frac{1}{2} \sum_{j=1}^{j=i}\left(\left|V_{r f, i}\right|-\frac{n V_{T}}{2} \ln \left(\frac{2 \pi\left|V_{r f, i}\right|}{n V_{T}}\right)\right)
$$

$V_{\text {out }, i}$ is the sum of all input contributions $\left|V_{r f, i}\right|$, and $V_{\text {term, } i}$ is defined as:

$$
V_{\mathrm{term}, i}=-\frac{n V_{T}}{2} \ln \left(\frac{2 \pi\left|V_{r f, i}\right|}{n V_{T}}\right)
$$

For high values of $V_{r f, i}$ for example $1 \mathrm{~V}, \mathrm{n}$ is equal to 1 and $V_{\text {term, } i}$ is equal to $0.07 \mathrm{~V}$, and therefore negligible compared to $\left|V_{r f, i}\right|$. Under these conditions, for values of $\left|V_{r f, i}\right|$ below $0.2 \mathrm{~V}, V_{\mathrm{out}, i}$ is expressed as Eq. (21) and for values above $0.2 \mathrm{~V}$ as:

$$
V_{\text {out }, i}=2 \sum_{j=1}^{j=i}\left|V_{r f, i}\right|
$$

Thus, $V_{\text {out }, i}$ is not affected by RF branches being active $\left(\left|V_{r f, i}\right| \mid \neq 0\right)$ or not $\left(\left|V_{r f, i}\right|=0\right)$. From Eq. (21), it is possible to express $V_{\text {out }, i}$ according to $V_{\text {out }, i-1}$ as shown by Eq. (22) and Eq. (23):

$$
\begin{aligned}
& V_{\text {out }, i}=2 \sum_{j=1}^{j=i-1}\left|V_{r f, i}\right|+2\left|V_{r f, i}\right| \\
& V_{\text {out }, i}=V_{\text {out }, i-1}+2\left|V_{r f, i}\right|
\end{aligned}
$$

Thus, $V_{\text {out }, i}$ is the sum of the output voltage $V_{\text {out }, i-1}$ and the amplitude of its input voltage $\left|V_{r f, i}\right|$. In the particular case where the amplitudes $\left|V_{r f, i}\right|$ are identical for each RF branch, it is possible to deduce that:

$$
V_{\text {out }, i}=i V_{\text {out }, i}
$$

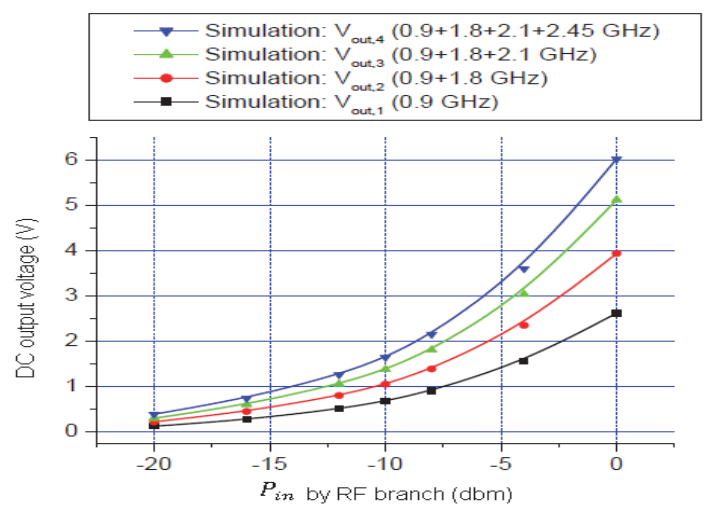

Figure $14 \mathrm{DC}$ output voltage simulated as a function of forward power for $1,2,3$ or 4 branches RF activated 
In Fig. 14 and Fig. 15, the simulation results show that the output voltage dc with the four active RF branches, $V_{\text {out } 4}$, is four times higher than the voltage obtained with only one active RF branch. The minimum input power to activate the rectenna is $-20 \mathrm{dBm}$, which is in the power range available in urban environments.

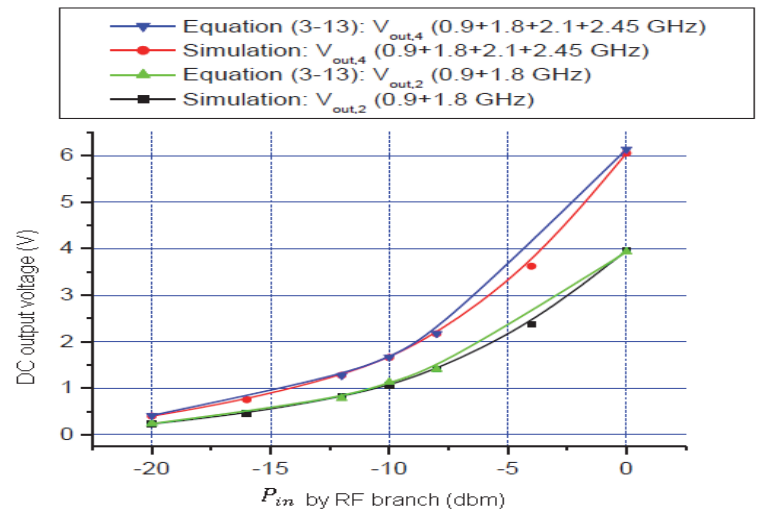

Figure 15 Simulated output voltage versus incident power for each frequency compared to the voltage calculated from the mathematical model for $R_{L}=11 \mathrm{k} \Omega$

\section{ENERGY MEASUREMENTS}

Practical measurements of RF power in specific RF bands in an urban and rural environment identify the predominant frequencies to be the GSM900 and GSM1800; however, the power density levels are relatively low. When placed between 50 to 250 meters from a GSM900 antenna, the measured RF power density varies between $1.2 \mathrm{~mW} / \mathrm{m}^{2}$ and $0.25 \mathrm{~mW} / \mathrm{m}^{2}$, and such variation (a function of distance) in the incident power will be experienced by the rectenna. Under these conditions, it may be necessary to add a DC-DC converter and an energy storage cell to the output of the rectenna to provide a constant DC supply voltage to the sensor.

\section{DISCUSSION AND CONCLUDING REMARKS}

This paper describes an innovative technique based on multiband RF energy recovery to recover the maximum energy in RF signals with a low level of power density. It discusses the key points for the design of a rectenna and analyses the architecture of the constituent blocks of the rectenna in detail.

It demonstrates the importance of the choice for the impedance matching network and the rectifier to provide optimum operation in a given environment. The study shows that energy recovery over several frequency bands is an interesting solution in an environment in which the amount of energy from separate RF sources is uncertain; having the ability to recover energy over a wide band provides flexibility to recover energy from the frequency bands present.

Two designs of multiband rectenna have been investigated. The first rectenna has been optimised to recover energy from three RF bands: GSM1800, UMTS and Wi-Fi. Simulation of the design shows that each RF band alone achieves an efficiency of $15 \%$ for a total input power of $-20 \mathrm{dBm}$. This efficiency reaches $25 \%$ with all three RF sources active and combined for a total incident power of $-20 \mathrm{dBm}$.
A second multiband rectenna to cover GSM900, GSM1800, UMTS and Wi-Fi was designed to take advantage of an improved architecture for the rectifier to combine the separate RF branches and improve efficiency and resilience to inactive RF bands. Investigation showed the effect of one branch RF inactive or having an output voltage lower than the other branches did not affect the total power for all bands together. Simulation results confirm the feasibility of powering small sensors with RF wave energy.

\section{REFERENCES}

[1] Harb, A. (2011). Energy harvesting: State-of-the-art. Renewable Energy, 36(10), 2641-2654. https://doi.org/10.1016/..renene.2010.06.014

[2] Le, T. T., Han, J., Von Jouanne, A., Mayaram, K., \& Fiez, T. S. (2006). Piezoelectric micro-power generation interface circuits. IEEE journal of solid-state circuits, 41(6), 14111420. https://doi.org/10.1109/JSSC.2006.874286

[3] Wang, W., Wang, N., Vinco, A., Siddique, R., Hayes, M., O'Flynn, B., \& O'Mathuna, C. (2013). Super-capacitor and thin film battery hybrid energy storage for energy harvesting applications. J. Phys. Conf. Ser, 476, 012105. https://doi.org/10.1088/1742-6596/476/1/012105

[4] Raghunathan, V. \& Chou, P. H. (2006). Design and power management of energy harvesting embedded systems. Proceedings of the 2006 international symposium on Low power electronics and design, 369-374. https://doi.org/10.1145/1165573.1165663

[5] Kansal, A., Hsu, J., Zahedi, S., \& Srivastava, M. B. (2007). Power management in energy harvesting sensor networks. ACM Transactions on Embedded Computing Systems (TECS), 6(4), 32-es. https://doi.org/10.1145/1274858.1274870

[6] Merabet, B., Cirio, L., Takhedmit, H., Costa, F., Vollaire, C., Allard, B., \& Picon, O. (2009, September). Low-cost converter for harvesting of microwave electromagnetic energy. 2009 IEEE Energy Conversion Congress and Exposition, 2592-2599. https://doi.org/10.1109/ECCE.2009.5316093

[7] Sun, H., Guo, Y. X., He, M., \& Zhong, Z. (2013). A dualband rectenna using broadband Yagi antenna array for ambient RF power harvesting. IEEE Antennas and Wireless Propagation Letters, 12, 918-921. https://doi.org/10.1109/LAWP.2013.2272873

[8] Breed, G. (2008). Improving the bandwidth of simple matching networks. High Frequency Electron, 7(56-60).

[9] Wong, A. C., McDonagh, D., Kathiresan, G., Omeni, O. C., El-Jamaly, O., Chan, T. C., \& Burdett, A. J. (2008). A 1V, micropower system-on-chip for vital-sign monitoring in wireless body sensor networks. 2008 IEEE International Solid-State Circuits Conference-Digest of Technical Papers, 138-602. https://doi.org/10.1109/ISSCC.2008.4523095

[10] Keysight Technologies. Advanced Design System (ADS). https://www.keysight.com/en/pc-1297113/advanceddesign-system-ads

[11] Hoel, V., Boret, S., Grimbert, B., Aperce, G., Bollaert, S., Happy, H., \& Cappy, A. (1999). 94-GHz low noise amplifier on InP in coplanar technology.

[12] Collado, A. \& Georgiadis, A. (2013). Conformal hybrid solar and electromagnetic (EM) energy harvesting rectenna. IEEE Transactions on Circuits and Systems I: Regular Papers, 60(8), 2225-2234. https://doi.org/10.1109/TCSI.2013.2239154

[13] Shinohara, N. \& Matsumoto, H. (1998). Experimental study of large rectenna array for microwave energy transmission. IEEE Transactions on Microwave Theory and Techniques, 46(3), 261-268. https://doi.org/10.1109/22.661713 
[14] Miura, T., Shinohara, N., \& Matsumoto, H. (2001). Experimental study of rectenna connection for microwave power transmission. Electronics and Communications in Japan (Part II: Electronics), 84(2), 27-36.

https://doi.org/10.1002/1520-6432(200102)84:2<27::AID-

ECJB4>3.0.CO;2-C

Contact information:

Okan OZGONENEL

(Corresponding author)

Ondokuz Mayis University,

Engineering Faculty, Electrical and Electronic Engineering Department,

55200 Atakum, Samsun, Turkey

E-mail: okanoz@omu.edu.tr

\section{Seda USTUN ERCAN}

Ondokuz Mayis University,

Engineering Faculty, Electrical and Electronic Engineering Department,

55200 Atakum, Samsun, Turkey

E-mail: sedau@omu.edu.tr 